\title{
Effect of Magnetic Impurities in a Two-Band Superconductor: A Point-Contact Study of Mn-Substituted $\mathrm{Mg} \mathrm{B}_{2}$ Single Crystals
}

\author{
R. S. Gonnelli, ${ }^{1}$ D. Daghero, ${ }^{1}$ G. A. Ummarino, ${ }^{1}$ A. Calzolari, ${ }^{1}$ M. Tortello, ${ }^{1}$ V. A. Stepanov, ${ }^{2}$ N. D. Zhigadlo, ${ }^{3}$ \\ K. Rogacki, ${ }^{3, *}$ J. Karpinski, ${ }^{3}$ F. Bernardini, ${ }^{4}$ and S. Massidda ${ }^{4}$ \\ ${ }^{1}$ Dipartimento di Fisica and CNISM, Politecnico di Torino, 10129 Torino, Italy \\ ${ }^{2}$ P.N. Lebedev Physical Institute, Russian Academy of Sciences, 119991 Moscow, Russia \\ ${ }^{3}$ Laboratory for Solid State Physics, ETHZ, CH-8093 Zurich, Switzerland \\ ${ }^{4}$ Dipartimento di Fisica, Università di Cagliari, 09042 Monserrato (CA), Italy \\ (Received 12 October 2005; published 19 July 2006)
}

\begin{abstract}
We present the first results of directional point-contact measurements in $\mathrm{Mg}_{1-x} \mathrm{Mn}_{x} \mathrm{~B}_{2}$ single crystals, with $x$ up to 0.015 and bulk $T_{c}$ down to $13.3 \mathrm{~K}$. The order parameters $\Delta_{\sigma}$ and $\Delta_{\pi}$ were obtained by fitting the conductance curves with the two-band Blonder-Tinkham-Klapwijk model. Both $\Delta_{\pi}$ and $\Delta_{\sigma}$ decrease with the critical temperature of the junctions $T_{c}^{\mathrm{A}}$, but remain clearly distinct up to the highest Mn content. Once analyzed within the Eliashberg theory, the results indicate that spin-flip scattering is dominant in the $\sigma$ band, as also confirmed by first-principles band-structure calculations.
\end{abstract}

PACS numbers: 74.50.+r, 74.45.+c, 74.70.Ad

The two-band character of superconductivity in $\mathrm{MgB}_{2}$ $[1,2]$ has been almost completely understood and explained by now, but the effects of disorder and chemical doping are still in need of some experimental clarification. Several substitutions have been tried [3] but none of the few successful have been able to enhance the $T_{c}$ of the compound. However, their study has proven useful to clarify the role of the different scattering channels and to try to control them selectively. Substitutions with magnetic impurities (Mn, Fe) represent a class of its own because of the spin-flip pair-breaking scattering that is expected to dramatically suppress superconductivity - even though the way it does it in a two-band superconductor has never been studied experimentally. Up until now, only two successful Mn substitutions in the $\mathrm{Mg}$ site have been reported: one in polycrystalline samples [4] and one in single crystals [5]. Here, we present the first results of point-contact spectroscopy (PCS) in the Mn-substituted $\mathrm{MgB}_{2}$ single crystals grown at ETHZ, which allowed us to study the effects of magnetic impurities on the order parameters (OPs) of a two-band superconductor. The trend of the OP amplitudes $\Delta_{\sigma}$ and $\Delta_{\pi}$ as a function of the critical temperature of the contacts, $T_{c}^{\mathrm{A}}$, can be explained within the Eliashberg theory as being mainly due to a doping-induced increase in the pair-breaking scattering within the $\sigma$ bands, with smaller contributions from the $\pi-\pi$ or the $\sigma-\pi$ channels. This result, apparently in contrast with the Mn position in the lattice, is however confirmed by first-principles calculations of the local effect of a Mn impurity on the band structure of $\mathrm{MgB}_{2}$.

The high-quality $\mathrm{Mg}_{1-x} \mathrm{Mn}_{x} \mathrm{~B}_{2}$ single crystals used for our measurements were grown by using the high-pressure technique set up for pure $\mathrm{MgB}_{2}$, and by replacing part of the $\mathrm{Mg}$ precursor with metallic Mn [5]. The Mn content $x$ of each crystal was measured by energy dispersive x-ray analysis (EDX) through a careful evaluation of the $\mathrm{Mn} / \mathrm{Mg}$ ratio; the crystals are single phase and homogeneous within $\pm \delta x(\delta x=0.0010)$. As shown elsewhere [5], the dependence of the lattice constants on the Mn content indicates that $\mathrm{Mn}$ replaces $\mathrm{Mg}$ in the lattice. The magnetic-field dependence of the Curie part $C^{*}$ of the magnetic moment clearly indicates that $\mathrm{Mn}$ ions are divalent (i.e., $\mathrm{Mn}^{2+}$ ) and in the low-spin state $(S=1 / 2)$, as also confirmed by our first-principles band-structure calculations. The crystals we used were carefully selected among those with the sharpest superconducting transitions and best structural properties, so that secondary phases or other impurities can be excluded. They had different $\mathrm{Mn}$ contents $x$ between 0.0037 and 0.0150 , corresponding to bulk critical temperatures $T_{c}$ (from dc magnetization) between 33.9 and $13.3 \mathrm{~K}$, with $\Delta T_{c}(10 \%-90 \%)$ increasing with doping from $0.65 \mathrm{~K}$ to $5.4 \mathrm{~K}$. The point contacts were made by putting a small drop of Ag paint $(\varnothing \simeq 50 \mu \mathrm{m})$ on the flat side surface of the crystal [6] so as to inject the current mainly parallel to the $a b$ planes, which in pure $\mathrm{MgB}_{2}$ is the best configuration to measure both the $\sigma$ - and $\pi$-band gaps [2,6]. In most cases, we studied the temperature and magnetic-field dependencies of the conductance curves $(d I / d V$ versus $V)$, so as to determine the critical temperature of the junction $\left(T_{c}^{\mathrm{A}}\right)$ and to understand whether one or two OPs were present $[6,7]$. The conductance curves were then normalized to the normal state and fitted with the two-band Blonder-Tinkham-Klapwijk (BTK) model [8], as described elsewhere [6]. The fitting function contains the OPs $\Delta_{\sigma, \pi}$, the coefficients $Z_{\sigma, \pi}$ (related to the potential barrier and to the Fermi-velocity mismatch at the interface), and the lifetime broadening coefficients $\Gamma_{\sigma, \pi}$ as adjustable parameters, plus the weight $w_{\pi}$ of the $\pi$-band contribution to the conductance. Whenever possible, we selected contacts with rather high resistance $\left(R_{\mathrm{N}} \gtrsim 30 \Omega\right)$, and with no dips [9] in the conductance curves, so as to fulfill the requirements for ballistic conduction [10].

Figure 1 reports some normalized conductance curves (symbols) in crystals with different Mn content. From now 
on, we will label the curves with the corresponding value of $T_{c}^{\mathrm{A}}$ instead of $x$ or the bulk $T_{c}$, since PCS is a local, surfacesensitive probe. $T_{c}^{\mathrm{A}}$ can be smaller than the bulk $T_{c}$ if the bulk transition is broad (as for the most-doped crystals) or if proximity effect occurs at the $S / N$ interface (when $\xi \approx$ $a$, being $a$ the true contact size).

Lines in Fig. 1 represent the two-band BTK fit of the experimental curves. In conventional superconductors, spin-flip scattering makes the gap become ill defined [11] or even creates bands of states within the original energy gap [12,13]. Strong-coupling calculations for $\mathrm{MgB}_{2}$ [14] have shown that magnetic impurities can give rise to subgap states in both the partial $\sigma$ - and $\pi$-band density of states (DOS), that are not taken into account by the BTK model. However, the subgap features in the DOS are by far smaller than the peaks connected to the OPs $\Delta_{\pi}$ and $\Delta_{\sigma}$ [14]. At finite temperature they are further smeared out, so that it would be very difficult to observe them experimentally. The same can be expected in the Andreev-reflection curves. In fact, all the experimental conductance curves are rather broadened and have smaller amplitude (see Fig. 1) than in pure $\mathrm{MgB}_{2}$ [6], so that even if subgap structures exist, they are practically unobservable. Hence, the BTK model can be used as a reasonable, first-order approxima-

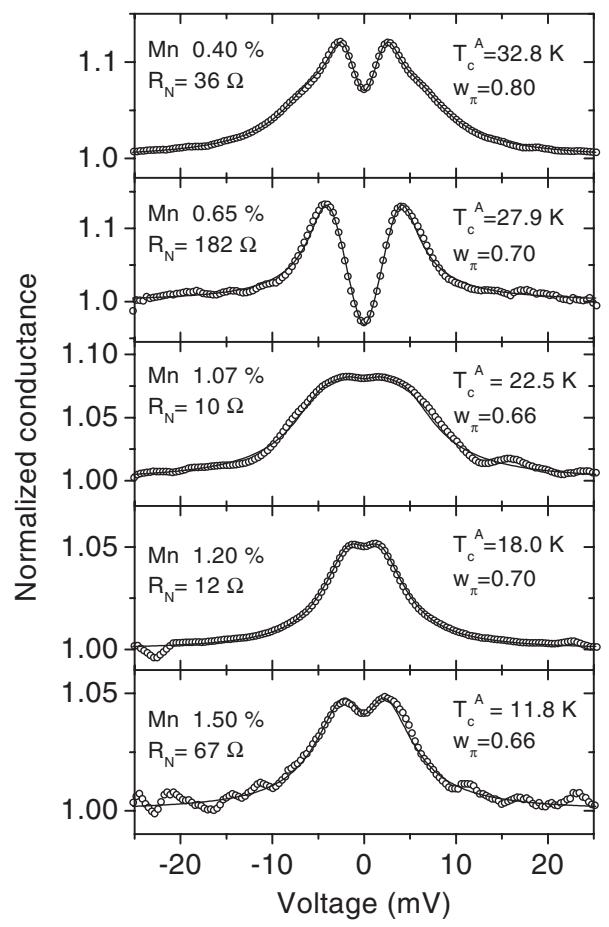

FIG. 1. Normalized conductance curves (symbols) of different $a b$-plane junctions in $\mathrm{Mg}_{1-x} \mathrm{Mn}_{x} \mathrm{~B}_{2}$ single crystals at $T=$ 4.2 K. The curves are labeled with the corresponding $T_{c}^{\mathrm{A}}$ values. The $\mathrm{Mn}$ content and the normal-state junction resistance are shown as well. Lines: best-fit curves given by the two-band BTK model. The $\pi$-band weight $w_{\pi}$ is also indicated. In the absence of specific theoretical predictions, we took $0.66 \leq w_{\pi} \leq 0.8$ as for $a b$-plane contacts in pure $\mathrm{MgB}_{2}[2,6]$. tion to a more specific model for Andreev reflection in a superconductor with magnetic impurities, which is lacking at the present moment.

Even if the fit of the curves in Fig. 1 indicates the existence of two OPs, it is clear that some curves-especially at the highest doping levels - show little or no structures associated to $\Delta_{\sigma}$. To check in a more convincing way if two OPs persist up to the highest Mn content, we studied the magnetic-field dependence of the $d I / d V$ curves in the whole range of $T_{c}^{\mathrm{A}}$.

Figure 2(a) shows the magnetic-field dependence of the conductance curve of a contact with $T_{c}^{\mathrm{A}}=31 \mathrm{~K}$ (circles) with the relevant two-band BTK fit (lines). The zero-field curve shows both the peaks corresponding to $\Delta_{\pi}$ and the smooth shoulders related to $\Delta_{\sigma}$. The magnetic field makes the small-gap features reduce in amplitude, progressively unveiling the underlying large-gap features. An outward shift of the peaks is observed at some $B=B^{*}$, when the $\sigma$-band structures become dominant and determine the shape of the curve. Unlike in pure $\mathrm{MgB}_{2}$, here $B^{*}$ is intense enough (if compared to $B_{c 2}$, strongly reduced by $\mathrm{Mn}$ impurities) to partly depress $\Delta_{\sigma}$, while the $\pi$-band partial conductance seems not to vanish at $B^{*}$. This makes it
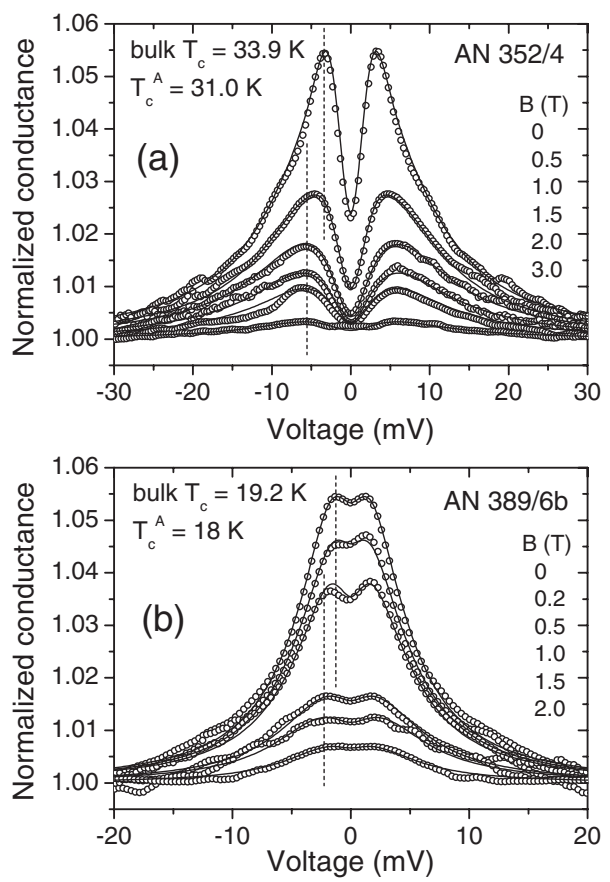

FIG. 2. Experimental conductance curves at $T=4.2 \mathrm{~K}$ of two contacts with $T_{c}^{\mathrm{A}}=31 \mathrm{~K}(\mathrm{a})$ and $T_{c}^{\mathrm{A}}=18 \mathrm{~K}(\mathrm{~b})$, in a magnetic field B $\| c$ (circles) and the relevant two-band BTK fit (lines). Vertical lines indicate the position of the conductance peaks. The best-fitting parameters in zero field are: $\Delta_{\pi}=2.5 \mathrm{meV}, \Delta_{\sigma}=$ $5.2 \mathrm{meV} ; \Gamma_{\pi}=2.3 \mathrm{meV}, \Gamma_{\sigma}=6.0 \mathrm{meV} ; Z_{\pi}=0.55, Z_{\sigma}=$ $0.82, w_{\pi}=0.7$ for panel (a); $\Delta_{\pi}=1.56 \mathrm{meV}, \Delta_{\sigma}=$ $2.8 \mathrm{meV} ; \Gamma_{\pi}=2.01 \mathrm{meV}, \Gamma_{\sigma}=3.8 \mathrm{meV} ; Z_{\pi}=0.43, Z_{\sigma}=$ $0.50, w_{\pi}=0.7$ for panel (b). The two-band BTK fit works well up to $B_{c 2}[\simeq 3.5 \mathrm{~T}$ in (a) and $\simeq 2.5 \mathrm{~T}$ in (b)]. 
impossible to separate the partial $\sigma$-and $\pi$-band conductances as we did in pure $\mathrm{MgB}_{2}$ [6,7], but the shift of the conductance peaks is anyway a clear proof of the existence of two OPs. The same happens in contacts with much lower $T_{c}^{\mathrm{A}}$ values. For example, Fig. 2(b) reports the magneticfield dependence of the normalized conductance curve of a contact with $T_{c}^{\mathrm{A}}=18 \mathrm{~K}$. Here the zero-field conductance does not show clear structures related to $\Delta_{\sigma}$ but an outward shift of the conductance maxima is observed anyway at $B=B^{*} \simeq 1 \mathrm{~T}$. For contacts with $T_{c}^{\mathrm{A}}<17 \mathrm{~K}$, the lowtemperature, zero-field conductance curves can be very well fitted with the two-band BTK model (see the bottom curve in Fig. 1), but the critical field is so small and the curves are so broadened that their magnetic-field dependence is not conclusive, at least within our experimental resolution. Moreover, some conductance curves in this region admit a standard (i.e., single-band) BTK fit as well, which gives an OP amplitude $\Delta$ that is the "average" of the values given by the two-band fit. However, the single-band fit often fails in reproducing both the position of the peaks and the width of the Andreev-reflection structures in the conductance, as shown in Fig. 3 for a contact with the lowest $T_{c}^{\mathrm{A}}$. This suggests that two OPs are likely to be present also in the most-doped samples.

The complete dependence of the OPs on $T_{c}^{\mathrm{A}}$ is reported in Fig. 4. Vertical error bars include both the uncertainty on the OP in each fit and the spread of OP values over different contacts with the same $T_{c}^{\mathrm{A}}$ (within the experimental uncertainty on $T_{c}^{\mathrm{A}}$ represented by horizontal error bars). For $T_{c}^{\mathrm{A}}<17 \mathrm{~K}$ only the results of the two-band BTK fit are shown, based on the preceding discussion and on the regular trend of the OPs for $T_{c}^{\mathrm{A}} \geq 18 \mathrm{~K}$. In fact, the presence of a single OP in the low- $T_{c}^{\mathrm{A}}$ region would imply a sudden change in the slope of the curves around $T_{c}^{\mathrm{A}}=$ $17 \mathrm{~K}$ that is not justified by any observed discontinuity in

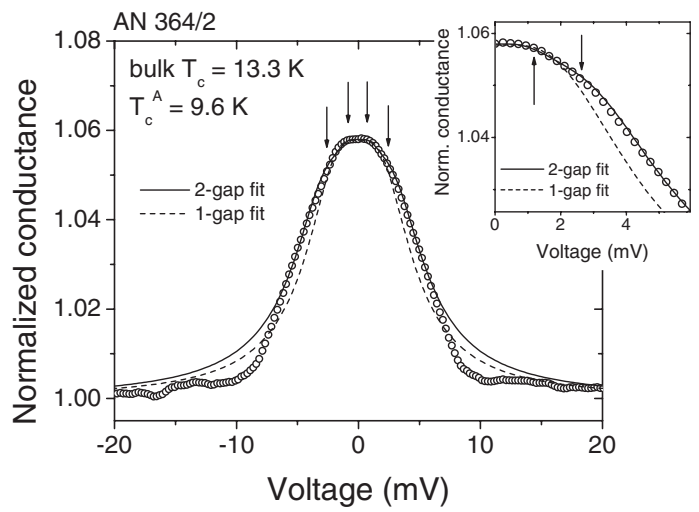

FIG. 3. Symbols: zero-field, low-temperature $(T=4.2 \mathrm{~K})$ conductance curve of a contact with $T_{c}^{\mathrm{A}}=9.6 \mathrm{~K}$ made on the most-doped crystals $(x=0.015)$. Solid line: best fit given by the two-band BTK model. Dashed line: best fit given by the standard (single-band) BTK model. Arrows indicate the smooth structures related to the two order parameters, which are magnified in the inset for clarity. the physical properties of the compound [5]. The persistence of two distinct OPs up to the highest $x$ value indicates that Mn doping does not significantly increase non-spinflip interband scattering. Actually, this is also suggested by the very low Mn concentration (1.5\% at most) and by the decrease of both the OPs with $T_{c}^{\mathrm{A}}$ [while interband scattering would increase $\Delta_{\pi}$ [15] ]. Changes in the DOSs at the Fermi level [15] and in the phonon spectra $\alpha^{2} F_{\sigma, \pi}(\omega)$ are certainly negligible here, because $\mathrm{Mn}$ is isovalent with $\mathrm{Mg}$ [5] and the Mn content is very small. The latter reason also allows neglecting the effect of $\sigma$ and $\pi$ intraband nonspin-flip scattering. This leads to conclude that the main possible cause of the experimental trend of Fig. 4 is an increase in the spin-flip scattering in the $\sigma-\sigma, \pi-\pi$, or $\sigma-\pi$ channels.

This simple picture can be made quantitative by solving the Eliashberg equations (EE) in the presence of randomly distributed magnetic impurities, treated within the Born approximation [16]. According to the reasoning above, we used the same phonon spectra, DOS values, and Coulomb pseudopotential as in pure $\mathrm{MgB}_{2}[2,6]$, and neglected all the non-spin-flip scattering rates. We thus took as the only adjustable parameters the spin-fip scattering rates within the bands $\left(\Gamma_{\sigma \sigma}\right.$ and $\left.\Gamma_{\pi \pi}\right)$ and between bands $\left(\Gamma_{\sigma \pi}\right)$. We immediately found that $\Gamma_{\sigma \sigma}$ is necessary to fit the $T_{c}^{\mathrm{A}}$ and OP values and that it must be greater than both $\Gamma_{\pi \pi}$ and $\Gamma_{\sigma \pi}$-otherwise $\Delta_{\pi}$ decreases too fast on decreasing $T_{c}^{\mathrm{A}}$. As for $\Gamma_{\sigma \pi}$, this agrees with the predictions of Ref. [14]. For simplicity, we analyzed separately the two cases: (a) $\Gamma_{\sigma \sigma}>\Gamma_{\pi \pi}, \Gamma_{\sigma \pi}=0$ and (b) $\Gamma_{\sigma \sigma}>\Gamma_{\sigma \pi}, \Gamma_{\pi \pi}=0$. First of all, we fixed $\Gamma_{\pi \pi}=\Gamma_{\sigma \sigma}=\Gamma_{\sigma \pi}=0$ at $T_{c}^{\mathrm{A}}=$ $39.4 \mathrm{~K}$ (i.e., $x=0$ ). Second, we found the values of the parameters that give the experimental values of $\Delta_{\sigma}$ and $\Delta_{\pi}$ at $T_{c}^{\mathrm{A}}=18.0 \mathrm{~K}$ [cases (a) and (b)], by solving the imaginary-axis EE and analytically continuing the solution to the real axis. Once determined the values of $\Gamma_{\pi \pi}$ and

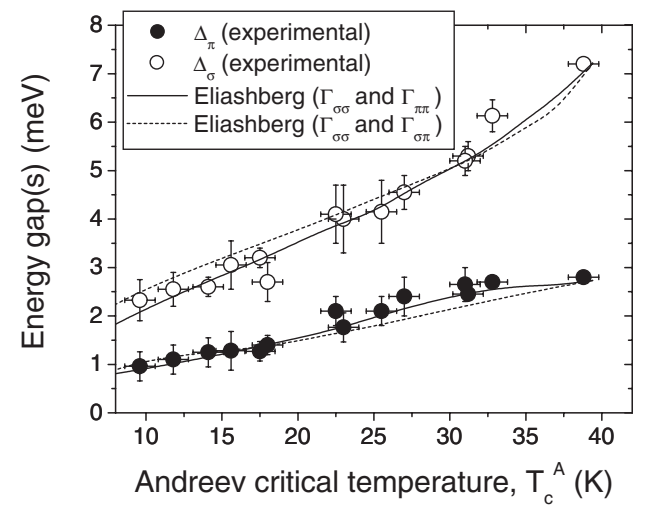

FIG. 4. Symbols: amplitudes of the order parameters as a function of the critical temperature of the junctions, $T_{c}^{\mathrm{A}}$. Lines: theoretical curves obtained by solving the Eliashberg equations in the presence of magnetic scattering in the $\sigma-\sigma$ and $\pi-\pi$ channels [case (a), solid lines] or in the $\sigma-\sigma$ and $\sigma-\pi$ channels [case (b), dashed lines]. 


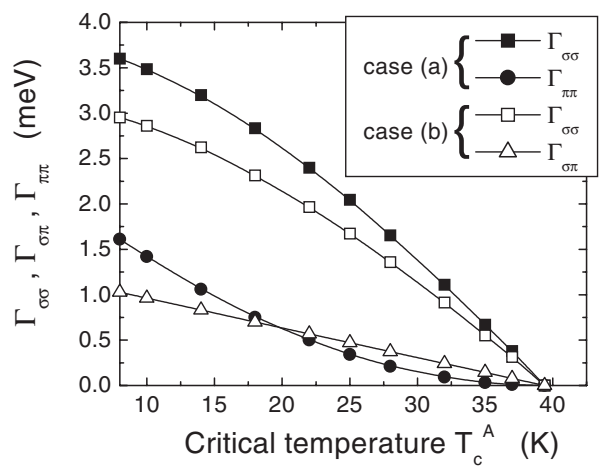

FIG. 5. Spin-flip scattering parameters used to fit the data of Fig. 4 as a function of $T_{c}^{\mathrm{A}}$. Solid symbols: case (a), $\Gamma_{\sigma \sigma}$ (squares) and $\Gamma_{\pi \pi}$ (circles). Open symbols: case (b), $\Gamma_{\sigma \sigma}$ (squares) and $\Gamma_{\sigma \pi}$ (triangles). Lines are guides to the eye.

$\Gamma_{\sigma \pi}$ in these two points, we searched for the simplest $\Gamma_{\pi \pi}\left(T_{c}^{\mathrm{A}}\right)$ and $\Gamma_{\sigma \pi}\left(T_{c}^{\mathrm{A}}\right)$ curves connecting them and allowing the fit of the experimental values of $T_{c}^{\mathrm{A}}$ and of the OPs in the whole doping range, with no restrictions on $\Gamma_{\sigma \sigma}$. We found out that these curves are a parabola (for $\Gamma_{\pi \pi}$ ) and a straight line (for $\Gamma_{\sigma \pi}$ ). In both cases, $\Gamma_{\sigma \sigma}$ follows an almost parabolic trend as a function of $T_{c}^{\mathrm{A}}$, as shown in Fig. 5. The resulting theoretical curves that best fit the OPs are reported in Fig. 4 as solid lines [case (a)] and dashed lines [case (b)]. The agreement between experimental data and theoretical calculations [especially in case (a)] is striking in the whole range of $T_{c}^{\mathrm{A}}$. That our simple but reasonable model works so well suggests that, up to $x=0.015$, $\mathrm{Mg}_{1-x} \mathrm{Mn}_{x} \mathrm{~B}_{2}$ can be treated as a perturbation of $\mathrm{MgB}_{2}$, with only the addition of $\sigma$ intraband magnetic scattering and smaller contributions from either the $\pi$ intraband or the interband magnetic scattering.

The intense Mn pair-breaking scattering in the $\sigma-\sigma$ channel has been recently predicted as being due to the hybridization of the $\sigma$ bands of $\mathrm{MgB}_{2}$ with the $d$ orbitals of Mn [17]. To prove that scattering in this channel is much greater than in the $\pi-\pi$ and $\sigma-\pi$ ones, we performed preliminary calculations of the electronic structure near a Mn impurity in a $2 \times 2 \times 4 \mathrm{MgB}_{2}$ superlattice. The results show that around the Fermi level $\left(E_{F}\right)$ there is a spin-down $d_{z^{2}}$ band, responsible for the Mn magnetic moment, very sensitive to the details of structural parameters [17]. The $\sigma$ and $\pi$ bands behave quite differently near the Mn impurity: while the $\pi$ electron spectral density is depleted around $E_{F}$ (due to the $\pi-d$ interaction), the $\sigma$ bands have a large amplitude. Furthermore, the $\sigma$ bands show a sizeable ( $\geq$ $15 \mathrm{meV}$ ) exchange splitting near $E_{F}$, larger than the superconducting gap-which is consistent with the observed complete suppression of superconductivity at about $2 \%$ of $\mathrm{Mn}$ [5]. On the other hand, the impurity-induced mixing of $\sigma$ and $\pi$ states around Mn (providing a qualitative indica- tion of interband scattering) is present but not very important. These results can explain the quick drop of $T_{c}$, with the persistence of the two distinct gaps, and a larger scattering within the $\sigma$ band [18].

In conclusion, we have presented the results of the first experimental study of the effects of magnetic impurities on the order parameters $\Delta_{\sigma}$ and $\Delta_{\pi}$ of a two-band superconductor. We have shown that, in $\mathrm{Mg}_{1-x} \mathrm{Mn}_{x} \mathrm{~B}_{2}, \Delta_{\sigma}$ and $\Delta_{\pi}$ decrease regularly with the critical temperature but remain clearly distinct down to the lowest $T_{c}^{\mathrm{A}}$. Within the Eliashberg theory, this is due to an increase in spin-flip scattering in the $\sigma$ bands on increasing the Mn content, with possible smaller contributions from the $\pi-\pi$ or the $\sigma-\pi$ channels. This somehow unexpected conclusion is also supported by first-principles calculations of the band structure of $\mathrm{MgB}_{2}$ in the vicinity of a $\mathrm{Mn}$ impurity.

This work was done within the PRIN Project No. 2004022024 and the INTAS Project No. 01-0617. V. A. S. acknowledges support by RFBR (Project No. 0602-16490).

*On leave from: Institute of Low Temperature and Structure Research, Polish Academy of Sciences, 50-950 Wroclaw, Poland.

[1] A. Y. Liu, I. I. Mazin, and J. Kortus, Phys. Rev. Lett. 87, 087005 (2001); H. J. Choi et al., Nature (London) 418, 758 (2002).

[2] A. Brinkman et al., Phys. Rev. B 65, 180517(R) (2002); A. A. Golubov et al., Phys. Rev. B 66, 054524 (2002).

[3] J. R. Cava, H. W. Zandbergen, and K. Inumaru, Physica (Amsterdam) 385C, 8 (2003).

[4] S. Xu, Y. Moritomo, K. Kato, and A. Nakimura, J. Phys. Soc. Jpn. 70, 1889 (2001).

[5] K. Rogacki et al., Phys. Rev. B 73, 174520 (2006).

[6] R. S. Gonnelli et al., Phys. Rev. Lett. 89, 247004 (2002).

[7] R.S. Gonnelli et al., Phys. Rev. B 69, 100504(R) (2004).

[8] G.E. Blonder, M. Tinkham, and T.M. Klapwijk, Phys. Rev. B 25, 4515 (1982).

[9] Goutam Sheet, S. Mukhopadhyay, and P. Raychaudhuri, Phys. Rev. B 69, 134507 (2004).

[10] A.M. Duif, A.G. M. Jansen, and P. Wyder, J. Phys. Condens. Matter 1, 3157 (1989).

[11] A. A. Abrikosov and L. P. Gor'kov, Sov. Phys. JETP 12, 1243 (1961).

[12] H. Shiba, Prog. Theor. Phys. 40, 435 (1968).

[13] E. Schachinger and J. P. Carbotte, Phys. Rev. B 29, 165 (1984).

[14] C. P. Moca and C. Horea, Phys. Rev. B 66, 052501 (2002).

[15] J. Kortus et al., Phys. Rev. Lett. 94, 027002 (2005).

[16] O. V. Dolgov et al., Phys. Rev. B 72, 024504 (2005).

[17] P. Jiji Thomas Joseph and Prabhakar P. Singh, cond-mat/ 0512675.

[18] F. Bernardini et al. (to be published). 